\title{
Sade sous le manteau : l'image du sadisme de Stendhal à Huysmans, entre mythe et figuration
}

\author{
Dominic Marion \\ University of Western Ontario
}

L'image du sadisme au XIX ${ }^{\mathrm{e}}$ siècle évolue dans un jeu de double contrainte (Bateson, 1956): si elle est dénoncée par les gardiens des belles-lettres et expulsée de la légitimité artistique, son influence sur les pratiques littéraires s'avère pourtant notable. Pour reprendre l'expression qu'utilise Anthony Glinoer dans son ouvrage sur l'éclosion en France des littératures dites frénétiques, Sade circule, autour des années 1820 et 1830, comme une « référence interdite » (2009, p. 50). 
Le roman-feuilleton à saveur criminelle se nourrit par exemple volontiers de l'ambivalence fébrile d'un public fasciné quoique craintif devant la représentation de la violence. L'emblème d'un tel engouement du lectorat pour le crime demeure sans doute le roman-feuilleton Les Mystères de Paris d'Eugène Sue, publié de 1842 à 1843 dans Le Journal des débats. Frédéric Soulié avait déjà exploité cette veine dans le même journal entre juin 1837 et mars 1838 avec Les Mémoires du diable, où la lecture de Justine est mise en scène dans le récit, le livre de Sade devenant le lieu même où la déperdition s'exprime: une jeune fille $\mathrm{y}$ rédige de son propre sang l'histoire de ses malheurs, intercalant les lignes imprimées d'une écriture manuscrite rouge (1858, p. 88-90). La première moitié du siècle semble de fait traversée par une certaine vogue sadienne, à partir de laquelle l'esthétisation du sang et de la mort imprègne autant la littérature sérieuse, ou «élitaire » (Glinoer, 2009, p. 24), que celle que Sainte-Beuve, en 1839, dénonce sous le qualificatif d'industrielle. Si le crime à sensation se vend à fort prix, s'il possède une indéniable force d'attraction qui révolutionne le marché des journaux et des cabinets de lectures, un important tabou marque toutefois la filiation qui s'établit avec Sade, dont le nom reste synonyme d'un profond interdit de publication.

J'entends ici retracer, dans le XIX ${ }^{\mathrm{e}}$ siècle français, le cadre d'une fascination entretenue par la littérature pour l'univers du morbide. Malgré le succès commercial et l'efficace publicitaire de la cruauté, la présence de Sade sur la scène littéraire demeure inavouable, brouillée par un masque qui se donne comme la condition de son influence. Cette présence fonctionne selon une étonnante absence référentielle à son texte: on évoque tout au plus Justine, si possible sans énoncer explicitement la référence. L'influence s'avère cependant secret 
de polichinelle: Jean-Marc Levent parle de la censure qui la traque comme d'une "consécration en creux » (2004, p. 175). Malgré ce que la critique sadienne laisse encore aujourd'hui trop souvent entendre, le XIX ${ }^{\mathrm{e}}$ siècle n'a pas oublié Sade. SainteBeuve fait à ce titre preuve d'une clairvoyance certaine, lorsqu'en 1843, il se permet d'identifier le romancier maudit sans le dénoncer aux autorités morales. Il s'abstient néanmoins de nommer les auteurs chez qui il relève une inspiration sadienne :

Il y a un fonds de De Sade masqué, mais non point méconnaissable, dans les inspirations de deux ou trois de nos romanciers les plus accrédités [...] j'oserai affirmer, sans crainte d'être démenti, que Byron et De Sade (je demande pardon du rapprochement) ont peut-être été les deux plus grands inspirateurs de nos modernes, l'un affiché et visible, l'autre clandestin, - pas trop clandestin. En lisant certains de nos romanciers en vogue, si vous voulez le fond du coffre, l'escalier secret de l'alcôve, ne perdez jamais cette dernière clé. (p.1314)

Il importe de reprendre cette clé que Sainte-Beuve tend au lecteur, non précisément pour ouvrir «le fond du coffre, l'escalier secret de l'alcôve », mais pour y attacher un certain fil rouge, qui, traversant le $\mathrm{XIX}^{\mathrm{e}}$ siècle français, permettra d'examiner comment, d'une part, la réception de Sade s'y esquisse à même une structure apparentée au mythe, et, d'autre part, cette réception consolide progressivement les conditions nécessaires à la formation d'une figure où pourra s'opérer un partage discursif entre la notion de sadisme, le nom propre de Sade, le corps biographique de l'homme et, enfin, le texte de l'écrivain. Nous évaluerons ainsi les effets de la fiction sadienne sur les pratiques discursives qui négocient le poids symbolique de son difficile héritage. 
Cette problématique s'ancrera d'abord chez Stendhal, à travers la représentation de la cruauté dans la nouvelle «Les Cenci », initialement publiée sans signature dans la Revue des Deux Mondes du 1er mars 1837. L'effusion de sang y rencontre la force d'évocation du mythe : restituant le récit historique de la violence perpétrée par François Cenci, Stendhal génère de fait un corps discursif qui se confronte au complexe de réception de l'histoire par la fiction. Nous déplacerons ensuite l'attention vers Huysmans afin de moduler dans un écart temporel cette réception de l'image du sadisme. Rédigeant une histoire de Gilles de Rais, le protagoniste du roman Là-bas, publié en 1891, réfléchit sur les conditions discursives de l'émergence d'une figure historique. Si le cas de Sade s'introduit dans le roman à partir de la mention explicite du sadisme, la substance même du projet romanesque s'avère bientôt hantée par un désir que la critique du $\mathrm{XX}^{\mathrm{e}}$ siècle viendra réaliser à travers l'élaboration de différentes figures de Sade, à commencer par le visage dithyrambique esquissé en 1909 par Guillaume Apollinaire dans son introduction à L'CEuvre du marquis de Sade.

\section{Stendhal et le pouvoir évocateur du mythe}

Afin d'évaluer le travail discursif qui a pu présider à l'approche interdite d'un matériau pourtant incontournable, il convient d'insister sur l'un des premiers textes qui sait interroger la problématique du sadisme de manière à saisir l'interdit du discours à même un discours sur l'interdit. Trop prudent pour se mesurer ouvertement à Sade, Stendhal apparaît comme un insigne représentant de cette veine, davantage sensible à l'épreuve pulsionnelle du sadisme qu'à sa valeur de scandale. La 
nouvelle «Les Cenci» mérite une attention particulière, notamment au niveau de sa stratégie narrative, qui cherche à ouvrir la figure de Don Juan sur le vague signifié de la criminalité associée au seigneur féodal.

Le contenu diégétique de la nouvelle se concentre autour de François Cenci, un riche citoyen de Rome à la fin du XVI siècle, qui se délecte de l'ignoble comportement qu'il entretient à l'égard de ses enfants. Il envoie ses fils à l'étranger, se plaît à leur refuser l'argent qui leur assurerait la subsistance la plus frugale, fait bâtir une église « dans la cour de son vaste palais près du Tibre » dans le seul but "d'avoir sous les yeux les tombeaux de tous ses enfants » (Stendhal, 1977, p. 249), et avec le désir de les y déposer lui-même. Il accable plus particulièrement sa fille Béatrix, avec qui il cherche à s'unir charnellement, qui plus est dans le lit conjugal et sous les yeux de sa femme Lucrèce. Confrontées à leur intolérable situation, la mère et la fille font assassiner leur bourreau. Leur crime finit par s'ébruiter et, malgré la compassion du public, elles sont conduites à l'échafaud.

Le lien qui autorise le rapprochement entre Sade et Stendhal s'annonce de prime abord plutôt ténu. S'il se forme ce qu'en 1979 Alain Goldschläger nomme « l'image sadienne dans l'œuvre de Stendhal ", cela tient davantage à la réfraction des enjeux liés à la formation discursive du mythe qu'à la référence directe à l'œuvre. Retracer l'héritage sadien au XIXe siècle exige ainsi de se replacer dans l'importante aura de scandale qui s'attache alors au nom de Sade et d'évaluer l'ampleur du compromis que l'opinion publique impose à l'écrivain qui, tout en voulant éviter de préjudicier sa diffusion publique, s'avère préoccupé par une filiation sadienne. 
Selon Goldschläger, Stendhal se présente en fait comme un «mauvais disciple de Sade » (2005). Fasciné par le pouvoir de subversion des procédés littéraires sadiens, Stendhal n'entend pas pour autant revendiquer explicitement son rapport à cet auteur controversé, qui ne recule devant aucun tabou et ose briser le carcan des mœurs les mieux établies. Les croisements thématiques sont pourtant majeurs : le meurtre et l'inceste sont après tout deux violations d'interdits que "Les Cenci » partage avec les plus violents récits sadiens. Mais, comme le souligne Goldschläger, «tous ces points de convergence révèlent davantage une communauté intellectuelle que littéraire, une réflexion sur un mythe et ses conséquences qu'une continuité » $(2005$, p. 194) : « bien plus que les textes », c'est «la perception sociale de la pensée novatrice [qui] joue » (p. 195). En somme, bien avant d'investir la problématique de la transgression à partir de la substance textuelle sadienne, c'est avec une image que Stendhal dialogue, celle que la formation du mythe cherche justement à conjurer. Goldschläger parle des «Cenci » comme un des textes de Stendhal «qui indiquent clairement la présence sadienne» (p.194); mais le lecteur y bute sur une très spécifique absence des signifiants "Sade » et "sadisme». Le nom de Sade apparaît toutefois dans les marginalia associées aux archives du texte, en une mention qui corrobore le rapprochement avec Cenci : «Ceci m'a l'air d'un roué passé au méchant à cause de son immense fortune, un de Sade » (p. 396).

Bien qu'il soit à peu près certain que Stendhal ait lu Sade, rien n'indique que sa lecture ait pu résister à l'amalgame que l'ère du temps façonne entre l'ordre de la fiction et la dépravation sexuelle dont l'époque accuse l'homme. Stendhal s'intéresse au pouvoir d'évocation d'une configuration 
libidinale noyée dans les dérives référentielles d'une logique apparentée au mythe. Si le lecteur de Stendhal peut sentir sourdre du texte l'aura du sadisme, ses éléments ne sont pourtant lisibles que sous le couvert d'une fiction historique. Ainsi, le délitement du sadisme dans le texte stendhalien ne repose pas tant sur la lisibilité spécifique de la figure de Sade que sur une récupération d'éléments subversifs, dont la narration se détache en situant la diégèse en Italie à la fin du XVIe siècle. Présentant l'horreur des faits, la narration évite toutefois d'en revendiquer la force subversive; elle se retranche plutôt derrière l'illusion de détachement que permet son traitement de la distance historique, amplifiant l'aspect mythique de l'horreur au lieu de la présenter comme une possibilité contemporaine. Mais c'est précisément là que Stendhal réussit à faire entrer son récit dans le champ d'attraction du mythe de Sade: en 1837, proposer au public français une nouvelle alliant violence passionnelle et mépris de la pudeur ne pouvait manquer d'attirer la puissance évocatrice du sadisme.

Le lecteur n'entre dans «Les Cenci » que pour aussitôt rencontrer la figure de Don Juan. La diégèse historique est en effet précédée d'une substantielle réflexion sur ce mythe, qui a notamment inspiré Molière et Mozart. D’entrée de jeu, Stendhal assimile le comportement de son protagoniste au donjuanisme :

Nous n'avons que deux portraits du don Juan d'Italie, tel qu'il dut se montrer, en ce beau pays, au XVI ${ }^{\text {e }}$ siècle, au début de la civilisation renaissante.

De ces deux portraits, il en est un que je ne puis absolument faire connaître, le siècle est trop collet monté. (1977, p. 239) 
Le second portrait est celui de François Cenci. Si, pour le premier, que Stendhal doit passer sous silence, Béatrice Didier pense à Borgia (Stendhal, 1977, p. 395), Claude Duchet se montre quant à lui catégorique : « il s'agit de Gilles de Rais, dont le nom, faut-il le rappeler, est si souvent associé à celui de Sade.» (1968, p. 237) L'on ne peut évidemment souscrire à cette méprise, qui fait de Gilles de Rais un « don Juan d'Italie ». Il faut pourtant reconnaître une certaine communication figurative qui semble avoir lieu chez Stendhal entre trois noms et que, suivant Duchet, l'on retrouve au cœur d'un puits mythologique :

Sade, Cenci, Rais : le cercle est bouclé et ces personnages hors format peuvent échanger leurs caractéristiques; dans les Chroniques, Cenci renvoie à Sade par les marginalia; et à Rais par prétérition, et Rais lui-même évoque Cenci, tout en reflétant Sade. (1968, p. 237-238)

Pour appuyer l'agencement elliptique proposé par Duchet, il convient de se reporter à un passage des Mémoires d'un touriste, où Stendhal dresse un portrait à la fois euphémistique et tendancieux de "Gilles de Retz [je respecte l'orthographe de l'édition citée] », qui y est en effet présenté comme un Don Juan, dont le caractère est d'emblée comparé à celui «du fameux François Cenci de Rome»: Rais aussi «mettait sa gloire à braver tout ce qu'on respecte» (1854, p. 331). Stendhal va jusqu'à ajouter que «cet homme singulier fut le premier de cette espèce » (p. 333). Sade ne recevra jamais le même traitement: Stendhal se refusera toujours à publier sous sa plume ce nom maudit, encore brûlant. Sur ce point, on pourrait dire qu'il s'incline devant la bonne conscience morale de son siècle bourgeois. Remarquons à ce titre la mise en garde sur laquelle se clôt le bref portrait qu'il donne de Gilles de Rais : 
« Il y aurait du danger à publier le procès de cet homme singulier. Dans ce siècle ennuyé et avide de distinctions, il trouverait peut-être des imitateurs. » (Stendhal, 1854, p. 337) Stendhal aurait peut-être pu dire la même chose de Sade : faute de le nommer, il se sera inspiré de son mythe naissant afin de souligner le potentiel subversif contenu dans le donjuanisme attribué à Cenci. Retracer l'ombre du sadisme telle qu'elle apparaît dans «Les Cenci » implique en ce sens d'investir la lettre à partir de ce qu'elle ne dit pas, ou plutôt de ce qu'elle dit sous le recouvrement du sadisme par le donjuanisme. Reconnu sous cette gestuelle de prétérition, le non-dit du sadisme stendhalien s'inscrit en faux dans le texte, à l'intersection de la cruauté et du très spécifique donjuanisme dont Stendhal revêt le personnage de François Cenci.

L'astuce figurative fonctionne de manière d'autant plus efficace que la popularité du mythe de Don Juan permet à Stendhal d'expliciter sans crainte de représailles la torsion qu'il fait subir au donjuanisme, pour subrepticement élargir sa théorie aux figures de Rais et de Sade : la nouvelle parle de François Cenci comme d'un «don Juan romain» qui «ne songeait aux autres hommes que pour marquer sa supériorité sur eux, s'en servir dans ses desseins ou les haïr » (Stendhal, 1977, p. 244). L'auteur tient néanmoins à ne pas assimiler tout à fait Cenci à la figure de Don Juan. Si le regard de Don Juan se mire inconditionnellement dans le regard de l'autre, Cenci va plus loin; il raffine son égoïsme vers une autocratie plus sadienne, où la médiation de l'autre ne reçoit qu'une fonction utilitaire :

Le don Juan n'a jamais de plaisir par les sympathies, par les douces rêveries ou les illusions d'un cœur tendre. Il lui faut, avant tout, des plaisirs qui soient des triomphes, qui puissent 
être vus par les autres, qui ne puissent être niés; il lui faut la liste déployée par l'insolent Leporello aux yeux de la triste Elvire.

Le don Juan romain s'est bien gardé de la maladresse insigne de donner la clef de son caractère, et de faire des confidences à un laquais, comme le don Juan de Molière; il a vécu sans confident, et n'a prononcé de paroles que celles qui étaient utiles pour l'avancement de ses desseins. Nul ne vit en lui de ces moments de tendresse véritable et de gaieté charmante qui nous font pardonner au don Juan de Mozart; en un mot, le portrait que je vais traduire est affreux. (p. 244; je souligne)

Cette "clef» du donjuanisme romain pourrait bien s'ouvrir sur une configuration libidinale à partir de laquelle la jouissance n'advient qu'à travers une violence perpétrée sur le corps d'autrui. Tout se passe chez Cenci comme si la «maladresse » du Don Juan de Molière était comblée par une adéquation se dessinant en filigrane entre le donjuanisme et la figure oblitérée de Sade. Cette perméabilité entre ces deux ordres figuratifs devient manifeste lorsque le narrateur parle $\mathrm{du}$ « triste rôle du don Juan pur » comme celui d'un sujet « qui ne cherche à se conformer à aucun modèle idéal, et qui ne songe à l'opinion du monde que pour l'outrager » (Stendhal, 1977, p. 246) : ce trait essentiel au donjuanisme stendhalien renoue avec l'obsession de la transgression qui anime le discours sadien. Lorsque le narrateur de la nouvelle parle « des libertins qui aiment la corruption pour elle-même, c'est-à-dire pour le plaisir de braver les opinions raisonnables de leurs contemporains» (p. 240), et qu'il soutient que «c'est à la religion chrétienne qu'[il] attribue la possibilité du rôle satanique de don Juan » (p. 240; je souligne), il devient évident que le texte cherche à prolonger la portée sociale du donjuanisme dans un corps de symptômes évoquant l'impiété sadienne. C'est là la mesure de la discrétion avec laquelle 
l'héritage de Sade s'actualise dans le corps des pratiques littéraires.

Les éléments sadiens que l'on trouve dans «Les Cenci » sont somme toute peu nombreux; ils sont souvent traités sur le mode de la litote, avec la virtuosité du voile que l'on reconnaît chez Stendhal. Lorsque le narrateur rapporte que «François Cenci était avide surtout d'événements bizarres qui pussent lui donner des peripezie di nuova idea, sensations nouvelles et inquiétantes » (Stendhal, 1977, p. 248), le rapprochement qui s'opère aux yeux du lecteur contemporain de Stendhal concerne les scandales qui méritèrent la prison à Sade, et non quelconque enjeu développé par le texte sadien. Cette désignation attire des référents qui s'agglomèrent sans manquer de faire communiquer les éléments d'un corps mythique à l'autre. Le tour de force orchestré par Stendhal consiste à faire palpiter silencieusement l'impulsion de la révolte à travers la maîtrise d'un idiome posé, davantage héritier d'un raffinement classique que de la fonction subversive de la littérature. Stendhal dessine là les contours d'une hétérogénéité qui correspond à la part de son œuvre qu'après Anthony Purdy l'on peut qualifier de «transgressive et sacrificielle» (1987, p. 400). Dans «Les Cenci », la représentation de la violence prend en charge une telle transgression sacrificielle. C'est à ce niveau que l'on peut reconnaître l'influence de Sade sur Stendhal, et ce, bien que ce dernier ne libère pas au grand jour toute l'ampleur de la violence sadienne: le fard de la langue recouvre la virulence par le défi plus convenu que Don Juan lance à la rigidité institutionnelle du mariage et de la famille.

Le lecteur voit s'éclaircir quelques traits, sans que pour autant se détache, perdue entre le cachot et l'interdit de lecture, 
l'image confuse qui irradie de la figure de Sade, toujours brouillée par la censure qui l'ostracise. Il s'en faut encore de beaucoup que la distinction puisse s'établir entre l'aura du sadisme et la biographie de Sade : le nom, le texte et la figure de l'écrivain ne se sont pas affranchis du tabou qui pèse sur eux, et ce dernier empêche leur présence culturelle d'échapper aux déformations factuelles propres au mythe.

\section{Huysmans et l'ouverture de la figure dans l'histoire}

Au milieu du XIXe siècle, la violence sadienne s'énonce encore à demi-mot dans le corps des pratiques littéraires; les circonvolutions et détours discursifs empruntés par les écrivains héritiers travaillent cependant à inscrire le contenu de la revendication sadienne dans l'espace culturel européen. Les plus précieux adjuvants à cette tâche comptent paradoxalement parmi ceux qui déforment le plus librement les faits à travers le traitement publicitaire: forte de la discrétion des gestes de référenciation, la critique littéraire de la première moitié du XIXe siècle émet des associations souvent abusives, qui viennent nourrir la formation du mythe Sade. La bonne conscience de la critique littéraire bourgeoise de 1834, incarnée par Jules Janin notamment, en vient de fait à soupçonner de sadisme toute violence apparemment investie d'une impulsion passionnelle. Mais, la plupart du temps, le rapport entre Sade et ce sadisme ne s'articule ni clairement ni surtout ouvertement.

Un imposant masque recouvre de fait ce qui pourrait s'énoncer comme figure de Sade, mais qui s'amalgame plutôt à la formation discursive du sadisme: le corps de l'homme se fond dans la scène de représentation du mythe. Une lourde 
confusion s'est installée entre l'orgie textuelle et l'orgie biographique, motivant par là la formation du néologisme « sadisme » (Boiste, 1834). Le cas de Sade préside bel et bien au développement d'une intuition figurative, mais celle-ci s'élabore en grande partie au détriment de la reconnaissance de l'écriture sadienne. C'est en ce sens qu'il faut parler d'une latence de la figure de Sade au XIX ${ }^{\text {e }}$ siècle: il en va d'une dissémination des éléments, des indices et des traces - tous foisonnants, doit-on préciser -, dont la réunion et la confrontation viendront plus tard extraire la pensée de Sade de l'image floue du sadisme.

Derrière l'interdit de lecture qu'elle soutient, l'écriture du corps mythologique de Sade cristallise en même temps une volonté de résistance contre l'hégémonie de l'autorité bourgeoise en matière de morale, volonté que l'on trouve dans le plus célèbre roman de Huysmans, paru en 1884. Une section d'À Rebours accorde en effet au sadisme une importante fonction subversive, qui témoigne qu'à travers la lecture baudelairienne de Sade, Huysmans semble avoir cherché à saisir le sadisme comme symptôme d'une infection sociale. À partir d'une réflexion sur Barbey d'Aurevilly, des Esseintes circonscrit explicitement «le sadisme, ce bâtard du catholicisme» (Huysmans, 1978a, p. 190), en tant qu'acte de transgression prisonnier de l'emprise de la religion. Le narrateur fait alors entendre l'expression «à rebours » dans le sens de la profanation du sacré catholique :

La force du sadisme, l'attrait qu'il présente, gît donc tout entier dans la jouissance prohibée de transférer à Satan les hommages et les prières qu'on doit à Dieu; il gît donc dans l'inobservance des préceptes catholiques qu'on suit même à rebours, en commettant, afin de bafouer plus gravement le Christ, les 
péchés qu'il a le plus expressément maudits : la pollution du culte et l'orgie charnelle.

$\mathrm{Au}$ fond, ce cas, auquel le marquis de Sade a légué son nom, était aussi vieux que l'Église; il avait sévi dans le XVIII siècle, ramenant, pour ne pas remonter plus haut, par un simple phénomène d'atavisme, les pratiques impies du sabbat au moyen âge. (p. 191)

Le narrateur revient au nom de Sade un peu plus loin :

Ce dégorgement d'impures railleries, de salissants opprobres était manifeste chez le marquis de Sade qui épiçait ses redoutables voluptés de sacrilèges outrages.

Il hurlait au ciel, invoquait Lucifer, traitait Dieu de méprisable, de scélérat, d'imbécile, crachait sur la communion, s'essayait à contaminer par de basses ordures une Divinité qu'il espérait vouloir bien le damner, tout en déclarant, pour la braver encore, qu'elle n'existait pas. (p. 192)

Sans se préoccuper de la situation d'énonciation de ces outrages, ce portrait d'un Sade blasphémateur attribue par ellipse le schéma d'une pratique discursive typique des libertins sadiens à l'homme qui les rédigeait. Ce n'est pas au niveau de la représentation du personnage historique qu'À Rebours innove; la conception du sadisme qui y est proposée intéresse bien davantage en ce qu'elle vient redessiner son image en tant que "phénomène d'atavisme », c'est-à-dire comme symptôme d'une maladie dont Sade ne serait que le dernier représentant insigne. En venant circonscrire le sadisme comme gestuelle sacrilège, l'hypothèse de l'athéisme à la gloire de Dieu dessine un certain seuil de figurabilité ${ }^{1}$ que le lecteur de Sade peut alors traverser

\footnotetext{
${ }^{1} \mathrm{Au}$ sens de Daniel Vaillancourt : « la figurabilité [...] veut redonner à la figure le mouvement de la pensée. Ce qui est figurable, ce qu'on peut figurer, signifie ce qui donne un horizon, comme le doigt qui, pointé vers le ciel, dessine une constellation en reliant les points-étoiles de la Grande Ourse. Dans la saisie d'un texte ou d'un document, il existe une invitation à penser, à l'élucidation
} 
et au-delà duquel apparaissent des traits qui délimitent les conditions d'émergence d'une figure. Les condensations du contenu mythique sont pourtant bien loin de se dissiper : elles survivront d'ailleurs tout au long du $\mathrm{XX}^{\mathrm{e}}$ siècle, telle une doxa inlassablement remaniée. À travers les thèmes chers à la Décadence, À Rebours n'entend pas ruiner le halo maudit qui entoure le nom de Sade; le roman participe toutefois à la progressive autonomisation de sa figure face à la pathologie individuelle du sadisme.

Sept ans après $\grave{A}$ Rebours, le roman Là-bas s'intéresse au sadisme en visitant les rapports historiquement tissés entre le satanisme et l'Église catholique. Le roman élabore son intrigue à l'aide de personnages tendus vers les extrêmes de la crise religieuse que Huysmans traverse alors : un sonneur de cloche exégète $\mathrm{du}$ rituel chrétien se dresse comme un rempart symbolique obsolète contre un prêtre présidant à de charnelles messes noires. Protagoniste du roman, l'écrivain Durtal travaille à la rédaction de l'histoire de Gilles de Rais; ce faisant, il est forcé de s'interroger sur les conditions d'émergence de la monstruosité sadique. La prise en charge du sadisme par Là-bas s'ouvre en quelque sorte là où Stendhal attribuait un rôle satanique au donjuanisme; c'est comme témoignage du diable que Durtal retrouvera le sens du sadisme de Rais. Le scandale Sade s'amenuise ici sous la continuité historique qui est créditée au sadisme. Alors qu'il résume à son ami le cadre de ses recherches, Durtal balaie du revers de la main le mythe de la monstruosité de l'homme Sade: parlant des viols et des

d'un objet qui fait cheminer et crée une perspective fondée sur du lacunaire : les points doivent être activés et reliés pour que la figure apparaisse. Quand elle paraît, elle surgit d'un seul coup, avant que tous les points ne soient nécessairement reliés. » (2012, p. 241) 
massacres d'enfants perpétrés par Rais, il soutient qu'à côté de cela «assurément, le marquis de Sade n'est qu'un timide bourgeois, qu'un piètre fantaisiste » (Huysmans, 1978b, p. 74). Rais apparaît ainsi autrement plus sadique que Sade.

Selon cette perspective, l'émergence discursive du sadisme à la fin du XVIII e siècle investit un contenu que la figure médiévale de Rais portait déjà, mais sans en dégager la spécificité libidinale. Le traitement de la figure de Rais par Huysmans suggère ainsi l'existence d'une certaine filiation reliant les meurtres du maréchal et ceux qui sont représentés dans l'œuvre de Sade. Afin de mettre en valeur la possibilité de cette filiation, il importe de s'attarder sur le processus de déformation populaire dont la figure de Rais a elle-même historiquement fait l'objet. À la fin du XIX siècle, l'abbé Eugène Bossard rapportait que les légendes et récits populaires « de l'Anjou, du Poitou et de la Bretagne » se référaient à l'histoire de Rais sous le couvert de la substitution de son nom par celui de Barbe-Bleue (1886, p. 399) : Bossard en déduisait que Rais était l'indéniable prototype de Barbe-Bleue. Sans pouvoir donner crédit à cette hypothèse, il faut cependant reconnaître la valeur de la collecte ethnologique faite par Bossard; celle-ci atteste que, dans les régions jadis habitées par le maréchal, la tradition orale a effectivement amalgamé l'histoire de Rais et la légende de Barbe-Bleue (1886, p. 383-425). Il ne s'agit pas de trancher ni de déterminer une fois pour toutes si Rais portait ou non la barbe. Dans la mesure où certains éléments des contes, mythes et légendes peuvent bénéficier d'une autonomie qui leur permet de migrer d'un récit à un autre, il importe plutôt de comprendre comment la teneur fantastique de la barbe du conte a pu s'intégrer à la construction de la figure historique. 
Comme Marc Soriano l'a montré, Charles Perrault semble bel et bien s'être inspiré d'une longue tradition orale afin de rédiger ses contes. Dans «La Barbe-Bleue», la barbe du personnage «le rendoit si laid \& si terrible, qu'il n'estoit ni femme ni fille qui ne s'enfuit de devant luy » (1697, p. 58); cela ne l'avait pas pour autant empêché de se marier à répétition. Suivant la logique circulaire de la temporalité mythologique, la terreur de l'auditeur du conte est d'entrée de jeu inscrite dans l'effort de personnification opéré par le récit : à la barbe revient la fonction de rendre le crime visible sur le visage de la légende; elle donne une version permanente du point d'horreur, dont la violence cadavérique s'avère pourtant explicitement énoncée par le texte de Perrault, lorsque la jeune mariée ouvre la porte du cabinet secret et qu'elle voit «que le plancher estoit tout couvert de sang caillé, \& que dans ce sang se miroient les corps de plusieurs femmes mortes, \& attachées le long des murs " (Perrault, 1697, p. 67-68). Cette description incarne le nœud traumatique qui condense dans un même signe l'histoire de divers trajets mythographiques. Forme signifiante, c'est aussi l'unité d'un contenu, d'une vision d'horreur qui hante l'inconscient de l'histoire tel qu'il est travaillé par la négociation des contraintes de souveraineté du pouvoir, de l'ascendant de l'Autre sur la vie et la mort du sujet. Dans sa réflexion sur le mythe, Roland Barthes soutient que la « répétition du concept à travers des formes différentes est précieuse pour le mythologue », précisément parce qu'elle "permet de déchiffrer le mythe: c'est l'insistance d'une conduite qui livre son intention » (1957, p.227). Suivant l'ordre sémiotique mythologique, la barbe criminelle du conte est ainsi attribuée à Rais, et ce, précisément dans la mesure où cette barbe est appelée à circuler de nouveau. 
Car de Rais à Sade, un lien de cruauté s'installe, qui voyage sur cette figure métonymique de la barbe. Dans l'une de ses apparitions sous la plume de Restif de La Bretonne, Sade devient «le vivodisséqueur, le même qui fut tiré de la Bastille avec une longue barbe blanche le 14 juillet 1789 » (1998, p. 19). Trois éléments mythologiques viennent ici se croiser dans le flot de la dénonciation. D'abord, on sait que Sade n'était plus à la Bastille le 14 juillet, ayant été transféré à Charenton dans la nuit du 3 au 4 juillet 1789, à une heure du matin, pour n'être libéré que le 2 avril 1790, à la suite de l'abolition des lettres de cachet (Lely, 1982, p. 378 et 433). En second lieu, la barbe ellemême apparaît comme une fantaisie résolument imaginaire : Jean-Jacques Pauvert souligne que Sade «n'a jamais eu de barbe ni blanche ni longue ni courte» (1998, p. 5). Enfin, la référence à la dissection s'avère fort probablement une déformation de l'affaire d'Arcueil, qui date de 1768 et qui se donne comme point d'ouverture historique du mythe de Sade. Lorsque Michel Delon parle de la réception publique de cette affaire, il note qu'une amplification mythologique semble s'emparer de chaque élément, au point où les récits qui circulent s'apparentent à l'univers du conte :

La présentation de l'affaire dérive vers de grands archétypes : Barbe-Bleue ou l'ogre dévorateur, Gilles de Rais ou le soldat par devoir changé en saigneur par volupté... Alors que Sade n'est encore que l'acteur d'un fait divers, son histoire est déjà littérature. Les fantasmes collectifs investissent son nom, gonflent les anecdotes, leur donne la dimension de mythe. (1990, p. XIV)

Serait-ce donc là le sens du repli de la souveraineté meurtrière de Rais / Barbe-Bleue sur le sadisme de Sade? La rumeur précédant la première publication de Justine en 1791 a certainement influencé les représentations que Restif accorde à 
Sade dans son œuvre. Il y a lieu de penser que, dans ce contexte mythographe, la barbe, fût-elle blanche, fonctionne comme le signe d'une cruauté meurtrière envers les femmes et qu'elle s'avère étroitement reliée à l'accusation de vivisection.

C'est donc de cet investissement fantasmatique des faits que Durtal, dans Là-bas, cherche à se défaire en dégageant la figure de Rais de la configuration confuse de son mythe. On remarquera que le projet de Huysmans s'appuie sur la parution alors récente du livre de l'abbé Bossard, dont la première édition remonte à 1885. Selon Durtal, c'est là « l'ouvrage le plus savant et le plus complet que l'on ait écrit sur le Maréchal » (Huysmans, 1978b, p. 176). Si Huysmans se fonde certainement sur ce livre pour construire les réflexions de Durtal, la réception de l'histoire par la fiction n'est pas que la redite improductive d'un contenu historique. En cherchant à retrouver à travers l'histoire de Rais le sens de la tradition subversive du satanisme, Durtal se débat de fait dans un dédale où sont intriquées les implications épistémologiques et figuratives inhérentes au repli du mythe de Barbe-Bleue sur Sade : il s'agit pour lui de dénouer ce point d'intersection où leurs figures se confondent dans la formation discursive du sadisme.

Ce désir de clarté, de renouveau du partage entre sadisme et savoir semble fonctionner dans le sens de la "rupture de l'écriture bourgeoise » dont Barthes parle dans Le Degré zéro de l'écriture (1953, p. 48-49), de cette "crise morale du langage littéraire» (1957, p. 243) qui a lieu au tournant des années 1850 en fonction du rejet par les écrivains français de «la fausse nature du langage littéraire 
traditionnel » (1957, p. 243). Barthes commentera plus tard l'ambition de son texte afin d'en souligner l'enjeu mythologique : «La subversion de l'écriture a été l'acte radical par lequel un certain nombre d'écrivains ont tenté de nier la littérature comme système mythique » (1957, p. 243). Porté par la représentation historicisante de Là-bas, le modelage de la conception du sadisme sur les meurtres commis par Rais se met en place à partir de la reconstruction d'une figure d'où l'image de Barbe-Bleue est expulsée. L'apparition de Sade dans le roman reste furtive; mais le geste épistémologique qui intéresse se confond avec la subsomption du sadisme sous l'idiome subversif du satanisme, ce qui marque la conquête d'une dissociation entre l'image du sadisme et le nom de Sade. Émerge alors un ordonnancement épistémologique qui commence à distinguer la vie de l'auteur de l'horreur des charges fictionnelles qu'il a produites.

\section{Anatomie biographique et dissection du symptôme}

Il aura fallu qu'émergent les documents, que se développe une rigueur critique minimale dans l'édition des textes, pour que s'opère au $\mathrm{XX}^{\mathrm{e}}$ siècle un partage entre le sadisme compris comme pathologie sexuelle, le nom propre de Sade et l'histoire biographique de l'homme, et pour qu'enfin le texte de l'écrivain émerge résolument en tant que symptôme d'un mal qui ne soit pas réductible à l'individualité de Sade. Là où il n'y a pas d'archive pour venir étayer le sens de la fiction, le sang de l'histoire ne coagule pas, et ses affects demeurent actifs dans l'inconscient de l'organisme social. 
Un certain circuit métonymique se dessine entre deux illustrations symptomatiques de l'évolution de la figure de Sade : de cette image de 1889 où Paul Cousturier représente Napoléon jetant Justine au feu, on passe au feu qui ronge la Bastille derrière la monumentale chair de pierre avec laquelle Man Ray représente Sade en 1938, dans ce fameux Portrait imaginaire. Au feu ne revient plus le même rôle symbolique : le déchet ne brûle plus au bûcher du pouvoir; il apparaît maintenant du côté d'une résistance

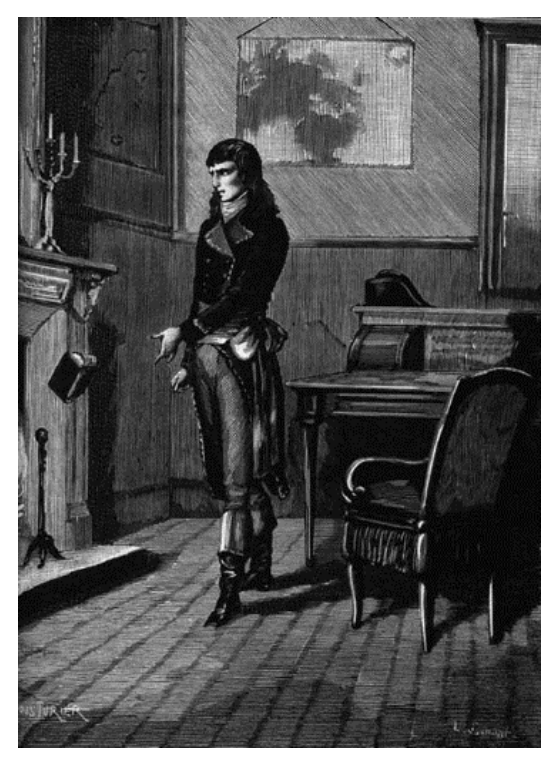
intemporelle contre la répression de la liberté individuelle. Même s'il conserve sa vocation destructrice, ravageant la Bastille devenu brasier, le feu s'avère toutefois impuissant contre la pierre massive du visage de Sade, dont l'image vient figurer la ténacité d'une vérité longtemps refoulée. La figure est lourde, claire, illuminée par le feu du désir souverain: la pierre devient le signifiant de la postérité sadienne.

[Napoléon Bonaparte jetant Justine au feu], gravure attribuée à Paul Cousturier, figurant dans le roman anonyme Le Marquis de Sade, ses aventures, ses œuvres, passions mystérieuses, folies érotiques, Paris, Fayard, 1885

Mais par-delà la figure d'un Sade de pierre, l'histoire de chair du dossier biographique ne sera jamais close. Les faits attestés suffisent cependant à justifier selon le droit moderne la criminalité de Sade: attacher et fouetter une femme non consentante tout en la menaçant de mort avec un couteau - 
c'est l'affaire d'Arcueil -, voilà de nos jours un cas bien assez substantiel pour condamner un homme. Une résistance, bien légitime en regard des faits biographiques, s'est toujours maintenue face à la vie que cet homme a menée. Seulement, cette résistance implique bien souvent un mouvement de régression, brouillant l'intellection du partage entre l'histoire biographique et la violence du texte. Que nous dit Michel Onfray, lorsqu'il prétend déconstruire le mythe de Sade? Sa position a d'abord le mérite d'identifier qu'au moment précis où le surréalisme a cru affranchir Sade du mythe, se constituait un renversement qui conservait de nombreuses caractéristiques mythologiques. Que le mythe ait perduré dans tous les efforts d'interprétation, jusqu'à ce qu'Onfray vienne rétablir les faits et dénoncer l'usurpatrice édification d'un Sade progressiste, libérateur et briseur de tabous, voilà ce dont le lecteur pourra juger à la mesure de la caricature qui lui est proposée : «Voilà en effet un homme qui a vécu comme un délinquant sexuel pendant toute son existence et qui a pratiqué ce qu'il théorisait » (Onfray, 2013, p. 121). Le point est bien sûr mieux appuyé qu'il y a deux cents ans, mais encore faut-il noter qu'Onfray réinvestit là une version du mythe qui dominait l'opinion publique du XIX ${ }^{\mathrm{e}}$ siècle.

Certes, on ne peut exclure d'emblée et sans appel la possibilité que Sade ait été criminel; qu'il ait même, comme le croit Marie-Laure Susini, disséqué des corps humains à La Coste (2004, p. 178). La valeur documentaire du symptôme de la fiction résiste toutefois à la catégorisation transitive de la vie de son auteur dans le cadre du réel historique. Pourrait-on prouver hors de tout doute que les ossements retrouvés dans le jardin de son château sont ceux de corps dépecés dont Sade aurait joui, cela ne révoquerait pas la valeur de symptôme portée par son écriture. 
La fiction sadienne parle d'une maladie sociale, d'un chancre qui ronge un monde qui n'en finit pas de traîner les avatars du régime féodal dans le mouvement de l'industrialisation généralisée. C'est cette indignation face à la jouissance du pouvoir qui est à l'origine des images de sang et de mort que nous avons ici analysées et qui nous permet de retracer dans le XIX ${ }^{\mathrm{e}}$ siècle sadien le cadre des violences que l'homme au pouvoir propage contre ses semblables. Appréhender l'extrémité de l'horreur permet de faire apparaître le symptôme, cancer émergeant au fil des opérations de dissection critique sur le corps de la fiction.

\section{Bibliographie}

ApollinAIRE, Guillaume. (1909), L'CEuvre du marquis de Sade, Paris, Bibliothèque des Curieux, coll. «Les Maîtres de l'amour ».

BARTHES, Roland. (1953), Le Degré zéro de l'écriture suivi de Nouveaux essais critiques, Paris, Seuil, coll. «Points Essais ».

—. (1957), "Le mythe, aujourd'hui », dans Mythologies, Paris, Seuil, coll. «Pierres vives », p. 213-268.

BATESON, Gregory, Don D. Jackson, Jay Haley et John Weakland (dir.). (1956), "Toward a Theory of Schizophrenia", Behavioral Science, vol. 1, no 4, p. 251-264.

BoISTE, Pierre Claude Victor et Charles Nodier. (1834), Dictionnaire universel de la langue française, avec le latin et les étymologies, 8 e éd., Paris, chez Lecointe et Pougin.

Bossard, Eugène. (1886), Gilles de Rais, Maréchal de France, dit Barbe-Bleue (1404-1440), 2e éd., Paris, Champion.

DELON, Michel. (1990), «Introduction », dans Sade, CEuvres I, Paris, Gallimard, coll. « Bibliothèque de la Pléiade», p. IX-LVIII. 
Duchet, Claude. (1968), «L'image de Sade à l'époque romantique ", dans Le Marquis de Sade, Paris, Armand Colin, p. 219-240.

GOLDSCHLÄGER, Alain. (1979), «L'image sadienne dans l'œuvre de Stendhal», Revue belge de philologie et d'histoire, t. 57, no 3, p. 612-627.

-. (2005), "Stendhal, mauvais disciple de Sade», L'Année Stendhalienne, no 4, Paris, Honoré Champion, p. 193-204.

GLINOER, Anthony. (2009), La Littérature frénétique, Paris, Presses universitaires de France, coll. "Les littéraires ».

HuYsmans, Joris-Karl. (1978a [1884]), À Rebours, éd. Pierre Waldner, Paris, Garnier-Flammarion.

—. (1978b [1891]), Là-bas, éd. Pierre Cogny, Paris, GarnierFlammarion.

Janin, Jules. (1834), «Le Marquis de Sade», Revue de Paris, tome douzième, p. 32-70.

LELY, Gilbert. (1982), Vie du marquis de Sade. Nouvelle édition revue et très augmentée, Paris, Pauvert/ Garnier, coll. «Époques ».

LEVENT, Jean-Marc. (2004) "Sade, l'homme naturel du XIXe siècle », Revue Lignes, nº 14 (Penser Sade), p. 167-191.

ONFRAY, Michel. (2013), "Sur un prétendu divin marquis... », dans Le Canari du nazi. Essais sur la monstruosité, Paris, Autrement, coll. « Universités populaires \& Cie», p. 117-125.

PERRAUlt, Charles. (1697), « La Barbe bleüe », dans Histoires ou Contes du temps passé: Avec des Moralitez, Paris, Claude Barbin, p. 57-82.

PURDY, Anthony (1987), «Stendhal et la crise de la valeur. De l'économie politique du signe », Cahiers d'histoire des littératures romanes, $\mathrm{n}^{\circ} 3 / 4$, p. 387-400. 
Restif DE LA BREtonne, Nicolas Edme. (1998 [1798]), L'AntiJustine ou Les délices de l'amour, Paris, La Musardine, coll. «Lectures amoureuses ».

SADE. ([1791] 1995), "Justine ou Les malheurs de la vertu ", dans CEuvres II, éd. Michel Delon, Paris, Gallimard, coll. «Bibliothèque de la Pléiade », p. 123-390.

SAINTE-BEuVE, Charles-Augustin. (1839), « De la littérature industrielle ", Revue des deux mondes, tome dix-neuvième, p. $675-691$.

-. (1843), "Quelques vérités sur la situation en littérature », Revue des deux mondes, tome troisième, p. 5-20.

SoRIano, Marc. (1977 [1968]), Les Contes de Perrault. Culture savante et traditions populaires, Paris, Gallimard, coll. « Tel ».

Soulié, Frédéric. (1858 [1837-1838]), Les Mémoires du diable, t. I, Paris, Michel Lévy frères.

STENDHAL. (1977 [1837]), «Les Cenci. $1599 »$, dans Chroniques italiennes, éd. Béatrice Didier, Paris, Garnier-Flammarion, p. 237-268.

—. (1854 [1838]), Mémoires d'un touriste, t. I, Paris, Michel Lévy frères.

SuE, Eugène. (1842-1843), Les Mystères de Paris, Paris, Charles Gosselin.

SuSINI, Marie-Laure. (2004), L'Auteur du crime pervers, Paris, Fayard.

VAILlAnCourT, Daniel. (2012), « La figure et ses fabriques. Tout ce qui est lu n'est pas figure ", dans Perspectives croisées sur la figure. À la rencontre du lisible et du visible, Montréal, Presses de l'Université du Québec, coll. «Approches de l'imaginaire », p. 235-251. 


\title{
Résumé
}

Cet article analyse les représentations littéraires du sadisme dans le cadre du XIXe siècle français. Si un héritage sadien se consolide dans la tradition littéraire de ce siècle, il n'en demeure pas moins que l'influence de Sade s'ancre souvent dans des textes qui omettent de mentionner son nom. C'est le cas de la nouvelle «Les Cenci », publiée par Stendhal en 1837. Dans Là-bas (1891), Huysmans, quant à lui, met en scène un protagoniste rédigeant l'histoire de Gilles de Rais. Contrairement au texte de Stendhal, qui sollicite l'aura du mythe afin de susciter l'intérêt du lecteur, Huysmans se positionne de manière critique par rapport aux conditions de formation du mythe. L'article retrace ainsi l'évolution générale que connaît la figure historique de Sade à travers le XIXe siècle, au sens où cette évolution est caractérisée par une mise à l'écart progressive des déformations mythologiques.

\begin{abstract}
This article addresses the literary representations of sadism during the $1^{\text {th }}$ century in France. While Sade's legacy is clearly consolidated in the literary tradition of this century, the fact remains that such influence is often anchored in texts that fail to mention Sade's name. Such is the case in the short story « Les Cenci », first published by Stendhal in 1837. In Là-bas (1891), Huysmans' protagonist is a writer preoccupied by the medieval murderer Gilles de Rais. Unlike Stendhal's text, which uses the aura of the myth in order to capture the interest of the reader, Huysmans is putting forward a critical approach regarding the conditions under which a myth is forming. This article therefore traces the general evolution experienced by the figure of Sade throughout the nineteenth century, an evolution that is characterized by a progressive elimination of mythological disformations.
\end{abstract}

